\title{
On Some Irregularity Measures of Graphs
}

\author{
I. Ž. Milovanović, E. I. Milovanović, V. Ćirić, N. Jovanović
}

\begin{abstract}
Let $\Gamma(G)$ be a set of all simple graphs of order $n$ and size $m$, without isolated vertices, with vertex degree sequence $d_{1} \geq d_{2} \geq \cdots \geq d_{n}>0$. A graph $G$ is regular if and only if $d_{1}=d_{2}=\cdots=d_{n}$. Each mapping $\operatorname{Irr}: \quad \Gamma(G) \mapsto[0,+\infty)$ with the property $\operatorname{Ir} r(G)=0$ if and only if $G$ is regular, is referred to as irregularity measure of graph. In this paper we introduce some new irregularity measures and inequalities that establish relations between them.
\end{abstract}

Keywords: Zagreb indices; irregularity measures; inequalities.

\section{Introduction}

We consider simple graphs $G=(V, E)$, where $V=\{1,2, \ldots, n\}$ and $E=\left\{e_{1}, e_{2}, \ldots, e_{m}\right\}$, without isolated vertices. Denote by $d_{1} \geq d_{2} \geq \cdots \geq d_{n}>0, d_{i}=d(i), i=1,2, \ldots, n$, a sequence of vertex degrees and by $d\left(e_{1}\right) \geq d\left(e_{2}\right) \geq \cdots \geq d\left(e_{m}\right)$, a sequence of edge degrees, whereby for each edge $e=\{i, j\} \in E$ holds $d(e)=d_{i}+d_{j}-2$. By $i \sim j$ we denote that vertices $i$ and $j$ are adjacent, while by $e_{i} \sim e_{j}$ that edges $e_{i}$ and $e_{j}$ are adjacent in a given graph $G$.

Let $\mathbf{A}$ be the adjacency matrix of $G$. Eigenvalues of $\mathbf{A}, \lambda_{1} \geq \lambda_{2} \geq \cdots \geq \lambda_{n}$ represent ordinary eigenvalues of graph $G$. Well known properties of these are (see for example $[6,30])$

$$
\sum_{i=1}^{n} \lambda_{i}=0 \quad \text { and } \quad \sum_{i=1}^{n} \lambda_{i}^{2}=\sum_{i=1}^{n} d_{i}=2 m
$$

Denote by $\mathbf{D}=\operatorname{diag}\left(d_{1}, d_{2}, \ldots, d_{n}\right)$ a diagonal matrix of vertex degrees in $G$. Then $\mathbf{L}=$ $\mathbf{D}-\mathbf{A}$ is the Laplacian matrix of $G$. The eigenvalues of $\mathbf{L}, \mu_{1} \geq \mu_{2} \geq \cdots \geq \mu_{n-1} \geq \mu_{n}=0$, are Laplacian eigenvalues of $G$. Some well known properties of Laplacian eigenvalues are

Manuscript received December 12, 2015; accepted March 1, 2016.

I. Ž. Milovanović, E. I. Milovanović and V. Ćirić are with the Faculty of Electronic Engineering, A. Medvedeva 14, 18000 Niš, Serbia; N. Jovanović is with the Faculty of Technical Sciences, K. Mitrovica, Serbia. 
$[6,10]$

$$
\sum_{i=1}^{n-1} \mu_{i}=\sum_{i=1}^{n} d_{i}=2 m \quad \text { and } \quad \sum_{i=1}^{n-1} \mu_{i}^{2}=\sum_{i=1}^{n} d_{i}^{2}+\sum_{i=1}^{n} d_{i}=M_{1}+2 m
$$

where

$$
M_{1}=M_{1}(G)=\sum_{i=1}^{n} d_{i}^{2}=\sum_{i \sim j}\left(d_{i}+d_{j}\right)
$$

is the first Zagreb index [24]. In the same paper, the second Zagreb index, $M_{2}$, is defined as

$$
M_{2}=M_{2}(G)=\sum_{i \sim j} d_{i} d_{j}
$$

The first and the second Zagreb indices of a given graph satisfy so called Zagreb indices inequality (see for example $[8,18]$ )

$$
\frac{M_{1}}{n} \leq \frac{M_{2}}{m}
$$

The first and second reformulated Zagreb indices, $E M_{1}$ and $E M_{2}$, are defined as [35, 44]

$$
E M_{1}=E M_{1}(G)=\sum_{i=1}^{m} d\left(e_{i}\right)^{2} \quad \text { and } \quad E M_{2}=E M_{2}(G)=\sum_{e_{i} \sim e_{j}} d\left(e_{i}\right) d\left(e_{j}\right) .
$$

A relation between Zagreb indices and reformulated Zagreb indices is established over the line-graph, $\mathscr{L}=\mathscr{L}(G)$, of the underlying graph $G$ (see $[6,10,30]$ ). Namely,

$$
E M_{1}(G)=M_{1}(\mathscr{L}(G)) \quad \text { and } \quad E M_{2}(G)=M_{2}(\mathscr{L}(G)) .
$$

It is not difficult to conclude that line-graph $\mathscr{L}(G)$ has $m$ vertices and $\frac{1}{2} M_{1}-m$ edges. If graph $G$ satisfies (1), then the following is valid

$$
\frac{E M_{1}}{2 m} \leq \frac{E M_{2}}{M_{1}-2 m} \quad \text { and } \quad \frac{E M_{1}}{n} \leq \frac{E M_{2}}{2 m-n}, \quad(2 m \neq n) .
$$

The forgotten topological index of $G$ is defined as [17, 22, 24, 45]

$$
F_{1}=\sum_{i=1}^{n} d_{i}^{3}=\sum_{i \sim j}\left(d_{i}^{2}+d_{j}^{2}\right)
$$

The equality that establishes a connection between graph invariants $M_{1}, M_{2}, E M_{1}$ and $F_{1}$ is given by [45]

$$
E M_{1}=F_{1}+2 M_{2}-4 M_{1}+4 m .
$$

More on these and other degree-based topological indices and their applications can be found in $[12,13,16,18,20,25,26,27,28,37,40,41,42,43]$. 
A graph $G$ is regular if and only if $d_{1}=d_{2}=\cdots=d_{n}>0$. Otherwise it is irregular. Let $\Gamma(G)$ be a set of all simple graphs of order $n, n \geq 2$, and size $m$, without isolated vertices. Each mapping $\operatorname{Irr}: \quad \Gamma(G) \mapsto[0,+\infty)$ with the property $\operatorname{Irr}(G)=0$ if and only if $G$ is regular, is referred to as irregularity measure of graph. There have been defined a lot of irregularity measures in the literature (see for example $[1,2,3,5,11,14,15,19,21,24,29$, $34])$. In the next section we mention some irregularity measures that are of interest for our work and introduce some new ones.

\section{Some irregularity measures of the graph}

Collatz and Sinogowitz [11] have proved that

$$
\lambda_{1} \geq \frac{2 m}{n}
$$

with equality holding if and only if $G$ is a regular graph. Using this inequality in [21] the Collatz-Sinogowitz irregularity measure was defined via

$$
\operatorname{Irr}_{C S}(G)=\frac{n \lambda_{1}}{2 m}-1
$$

Nikiforov [34] introduced irregularity measure referred to as degree deviation

$$
S(G)=\sum_{i=1}^{n}\left|d_{i}-\frac{2 m}{n}\right| .
$$

We will call this measure Nikiforov irregularity measure and denote it by $\operatorname{Ir}_{N}(G)$.

Bell [5] considered the variance of vertex degrees as irregularity measure. It is defined as

$$
\operatorname{VAR}(G)=\frac{1}{n} \sum_{i=1}^{n}\left(d_{i}-\frac{2 m}{n}\right)^{2}
$$

It is not difficult to see that both $\operatorname{Irr}_{N}(G)$ and $\operatorname{VAR}(G)$ are special case of the following graph invariant

$$
R_{\alpha}(G)=\left(\frac{1}{n^{\alpha-1}} \sum_{i=1}^{n}\left|d_{i}-\frac{2 m}{n}\right|^{\alpha}\right)^{1 / \alpha}, \quad \alpha \geq 1 .
$$

Hamzeh and Reti [24] defined the following irregularity measure

$$
\operatorname{IR} M_{1}(G)=M_{1}-\frac{4 m^{2}}{n} .
$$

Edwards [14] considered the value $C_{\gamma}$ as irregularity measure, defined as

$$
1+C_{\gamma}^{2}=\frac{n M_{1}}{4 m^{2}}
$$


whereby $C_{\gamma}^{2}$ is a real number. He proved that $C_{\gamma}=0$ if and only if $G$ is regular. It is not difficult to see that the following relation between $\operatorname{VAR}(G), \operatorname{IRM}(G)$ and $C_{\gamma}^{2}$ holds

$$
C_{\gamma}^{2}=\frac{n^{2}}{4 m^{2}} \operatorname{VAR}(G)=\frac{n}{4 m^{2}} \operatorname{IRM}(G) .
$$

Therefore these measures can be considered as equivalent. Since $C_{\gamma}^{2} \geq 0$, according to (4) it follows that

$$
M_{1} \geq \frac{4 m^{2}}{n} .
$$

Equality holds if and only if $G$ is regular. More on the inequality (5) one can find in [12, $20,25,44]$. Based on (5), in [21] Edwards' irregularity measure was defined as

$$
\operatorname{Irr}_{E}(G)=\sqrt{\frac{n M_{1}}{4 m^{2}}-1} .
$$

Ilić and Stevanović [25] (see also [45]) proved the following inequality

$$
M_{2} \geq \frac{4 m^{3}}{n^{2}},
$$

with equality holding if and only if $G$ is a regular graph. Based on this, a new irregularity measure, Ilić-Stevanović measure, can be defined as

$$
\operatorname{Irr}_{I S}(G)=\sqrt[3]{\frac{n^{2} M_{2}}{4 m^{3}}-1} .
$$

In [24] the following irregularity measure was proposed

$$
I R M_{2}(G)=M_{2}-\frac{4 m^{3}}{n^{2}} .
$$

Since

$$
\operatorname{IRM}_{2}(G)=\frac{4 m^{3}}{n^{2}} \operatorname{Irr}_{I S}(G),
$$

it follows that $I R M_{2}(G)$ is not different from the $\operatorname{Irr}_{I S}(G)$.

From the inequality (5) the following inequality can be directly derived [13]

$$
E M_{1} \geq \frac{\left(M_{1}-2 m\right)^{2}}{m} \geq \frac{4 m(2 m-n)^{2}}{n^{2}},
$$

with equality holding if and only if $G$ is regular. Based on this inequality, another irregularity measure can be defined as

$$
\operatorname{Irr}_{1}(G)=\sqrt[3]{\frac{n^{2} E M_{1}}{4 m(2 m-n)^{2}}-1}, \quad 2 m \neq n .
$$


In [26] (see also [13]) based on inequality (6) the following one was proved

$$
E M_{2} \geq \frac{\left(M_{1}-2 m\right)^{3}}{2 m^{2}}
$$

with equality holding if and only if $G$ is regular. According to this and the inequality (5), it follows that

$$
E M_{2} \geq \frac{4 m(2 m-n)^{3}}{n^{3}} .
$$

Now we can define Ilić-Zhou irregularity measure

$$
\operatorname{Irr}_{I Z}(G)=\sqrt[4]{\frac{n^{3} E M_{2}}{4 m(2 m-n)^{3}}-1}, \quad 2 m \neq n
$$

Denote by $F_{1}^{(\alpha)}=\sum_{i=1}^{n} d_{i}^{\alpha}$, where $\alpha(\alpha \geq 1)$ is a real number. It is not difficult to see that $F_{1}^{(1)}=2 m, F_{1}^{(2)}=M_{1}$ and $F_{1}^{(3)}=F_{1}$. According to the Chebyshev's inequality (see for example [31])

$$
\sum_{i=1}^{n} p_{i} \sum_{i=1}^{n} p_{i} a_{i} b_{i} \geq \sum_{i=1}^{n} p_{i} a_{i} \sum_{i=1}^{n} p_{i} b_{i}
$$

for $p_{i}=b_{i}=d_{i}$ and $a_{i}=d_{i}^{\alpha-2}, \alpha \geq 2$, inequality (7) becomes

$$
\sum_{i=1}^{n} d_{i}^{\alpha} \geq \frac{M_{1}}{2 m} \sum_{i=1}^{n} d_{i}^{\alpha-1} .
$$

After iterating the above inequality, we obtain

$$
F_{1}^{(\alpha)}=\sum_{i=1}^{n} d_{i}^{\alpha} \geq \frac{\left(M_{1}\right)^{\alpha-1}}{(2 m)^{\alpha-2}}, \quad \alpha \geq 2 .
$$

Based on this and inequality (5), we have that

$$
F_{1}^{(\alpha)}=\sum_{i=1}^{n} d_{i}^{\alpha} \geq \frac{(2 m)^{\alpha}}{(n)^{\alpha-1}}, \quad \alpha \geq 2,
$$

whereby the equality holds if and only if $G$ is regular. This inequality enables us to define a bunch of irregularity measures for various $\alpha$

$$
\operatorname{Irr}^{(\alpha)}(G)=\left(\frac{n^{\alpha-1} F_{1}^{(\alpha)}}{(2 m)^{\alpha}}-1\right)^{1 / \alpha}
$$


It is obvious that $\operatorname{Ir} r^{(2)}(G)=\operatorname{Irr}_{E}(G)$. Also, the following might be an interesting irregularity measure when $\alpha=3$

$$
\operatorname{Irr} r^{(3)}=\sqrt[3]{\frac{n^{2} F_{1}}{8 m^{3}}-1}
$$

Remark 1 Let us note that equivalent irregularity measure to the above was defined in [38].

In [19] Goldberg noticed that the simplest irregularity measure is

$$
d(G)=d_{1}-d_{n}
$$

We will call this measure Goldberg's irregularity measure, and consider it in the form

$$
\operatorname{Irr}_{g}(G)=\frac{d_{1}}{d_{n}}-1
$$

Remark 2 It is obvious that a lot of irregularity measures can be defined. The question is do we really need all of them? From each of them we can conclude whether a given graph is regular or not, but if we are interested to see how much the considered graph deviates from the regular one, the differences arise. It is desirable that irregularity measure is sensitive to the changes of all basic graph parameters: $n, m, d_{1}$ and $d_{n}$. Thus, for example Golberg's irregularity measure is really simple, but it is (completely) insensitive to the changes of parameter $m$ when parameters $d_{1}$ and $d_{n}$ remain unchanged. Thus, for example, if graph $G_{1}$ is obtained by adding edges to the graph $G$, while $d_{1}$ and $d_{n}$ remain unchanged, then $\operatorname{Irr}_{g}\left(G_{1}\right)=\operatorname{Irr}_{g}(G)$. However, it is obvious that $G$ deviates from regularity more than $G_{1}$. Also, the measure $\operatorname{Irr}(G)=\frac{2 m}{n d_{n}}-1,\left(d_{n} \neq 0\right)$, is not sensitive to the changes of parameter $d_{1}$ when $n, m$ and $d_{n}$ are unchanged. Nevertheless, these "simple" irregularity measures are useful for determining bounds of other irregularity measures or when comparing different measurs.

\section{Inequalities for irregularity measures}

In this section we prove some inequalities between irregularity measures defined in the previous section. Also we establish bounds for some of them. But, first we recall some results from the literature needed for our work.

In [19] Goldberg proved the following inequality

$$
\operatorname{Irr}_{C S}(G) \geq \frac{4 m^{2}}{n^{2}} \sqrt{\frac{2 m}{n d_{1}}} \operatorname{Irr}_{E}(G)^{2}
$$


Nikiforov [34] proved the following inequalities

$$
\frac{\sqrt{2 m}}{2 n} \operatorname{Irr}_{E}(G)^{2} \leq \operatorname{Irr}_{C S}(G) \leq \frac{n \sqrt{\operatorname{Irr}_{N}(G)}}{2 m},
$$

and

$$
\frac{\operatorname{Irr}_{N}(G)^{2}}{4 n m \sqrt{2 m}} \leq \operatorname{Irr}_{C S}(G) \leq \sqrt{\frac{n^{2}}{2 m} \operatorname{Irr}_{E}(G)} .
$$

Based on the Popovicu [36] and Nagy [33] inequalities (see also [39]) the following inequalities can be obtained

$$
\frac{\sqrt{n} d_{n} \operatorname{Irr}_{g}(G)}{2 \sqrt{2} m} \leq \operatorname{Irr}_{E}(G) \leq \frac{n d_{n} \operatorname{Irr}_{g}(G)}{4 m} .
$$

In the following theorem we improve the right-hand side of inequality (13).

Theorem 1 For each graph $G, G \in \Gamma(G)$, the following is valid

$$
\operatorname{Irr}_{E}(G) \leq \frac{\sqrt{\alpha(n)} n d_{n} \operatorname{Irr} r_{g}(G)}{2 m},
$$

where

$$
\alpha(n)=\frac{1}{n}\left\lfloor\frac{n}{2}\right\rfloor\left(1-\frac{1}{n}\left\lfloor\frac{n}{2}\right\rfloor\right) .
$$

Equality holds if and only if $G$ is regular.

Proof. For real numbers $p_{1}, p_{2}, \ldots, p_{n}, a_{1}, a_{2}, \ldots, a_{n}$ and $b_{1}, b_{2}, \ldots, b_{n}$ with the property

$$
p_{i} \geq 0, \quad 0<r_{1} \leq a_{i} \leq R_{1}<+\infty, \quad 0<r_{2} \leq b_{i} \leq R_{2}<+\infty, \quad i=1,2, \ldots, n
$$

Andrica and Badea [4] proved the following inequality

$$
\left|\sum_{i=1}^{n} p_{i} \sum_{i=1}^{n} p_{i} a_{i} b_{i}-\sum_{i=1}^{n} p_{i} a_{i} \sum_{i=1}^{n} p_{i} b_{i}\right| \leq\left(R_{1}-r_{1}\right)\left(R_{2}-r_{2}\right) \sum_{i \in S} p_{i}\left(\sum_{i=1}^{n} p_{i}-\sum_{i \in S} p_{i}\right),
$$

where $S$ is a subset of the set $I_{n}=\{1,2, \ldots, n\}$ for which the value

$$
\left|\sum_{i \in S} p_{i}-\frac{1}{2} \sum_{i=1}^{n} p_{i}\right|
$$

reaches a minimum. Let $S=\{1,2, \ldots, k\}, 1 \leq k \leq n$, and $p_{i}=1$, for $i=1,2, \ldots, n$. Then, according to (17) $k=\left\lfloor\frac{n}{2}\right\rfloor$, i.e. $S=\left\{1,2, \ldots,\left\lfloor\frac{n}{2}\right\rfloor\right\}$. Now, for $S=\left\{1,2, \ldots,\left\lfloor\frac{n}{2}\right\rfloor\right\}, p_{i}=1$, $a_{i}=b_{i}=d_{i}, i=1,2, \ldots, n, R_{1}=R_{2}=d_{1}$ and $r_{1}=r_{2}=d_{n}$ according to (16) we have that

$$
n \sum_{i=1}^{n} d_{i}^{2}-\left(\sum_{i=1}^{n} d_{i}\right)^{2} \leq\left(d_{1}-d_{n}\right)^{2}\left\lfloor\frac{n}{2}\right\rfloor\left(n-\left\lfloor\frac{n}{2}\right\rfloor\right)
$$


i.e.

$$
n M_{1}-4 m^{2} \leq\left(d_{1}-d_{n}\right)^{2}\left\lfloor\frac{n}{2}\right\rfloor\left(n-\left\lfloor\frac{n}{2}\right\rfloor\right) .
$$

The above inequality can be rewritten in the form

$$
4 m^{2}\left(\frac{n M_{1}}{4 m^{2}}-1\right) \leq n^{2} d_{n}^{2}\left(\frac{d_{1}}{d_{n}}-1\right)^{2} \frac{1}{n}\left\lfloor\frac{n}{2}\right\rfloor\left(n-\left\lfloor\frac{n}{2}\right\rfloor\right)
$$

wherefrom we obtain the result of the theorem.

Equality in (18) holds if and only if $d_{1}=d_{2}=\cdots=d_{n}$, so the equality in (14) holds if and only if $G$ is a regular graph.

\section{Remark 3 Since}

$$
\alpha(n)=\frac{1}{n}\left\lfloor\frac{n}{2}\right\rfloor\left(1-\frac{1}{n}\left\lfloor\frac{n}{2}\right\rfloor\right)=\frac{1}{4}\left(1-\frac{(-1)^{n+1}+1}{2 n^{2}}\right)= \begin{cases}\frac{1}{4}, & \text { if } n \text { is even } \\ \frac{(n-1)(n+1)}{4 n^{2}}, & \text { if } n \text { is odd }\end{cases}
$$

the inequality (14) is stronger than the right-hand side of inequality (13) for odd $n$.

Corollary 1 For each graph $G, G \in \Gamma(G)$, the following inequality is valid

$$
\operatorname{Irr}_{C S} \leq \frac{n}{2 m} \sqrt{\sqrt{\alpha(n)} n d_{n} \operatorname{Irr}_{g}(G)} .
$$

Equality holds if and only if $G$ is a regular graph.

In inequalities (11) and (12) indirect relationships between irregularity measures $\operatorname{Irr}_{N}(G)$ and $\operatorname{Irr}_{E}(G)$ are given. In the following theorem we prove the inequality that establishes direct connection between these irregularity measures.

Theorem 2 For each graph $G, G \in \Gamma(G)$, the following is valid

$$
\frac{2 \sqrt{2} m}{\sqrt{n}} \operatorname{Irr}_{E}(G) \leq \operatorname{Irr}_{N}(G) \leq 2 m \operatorname{Irr}_{E}(G) .
$$

Equality holds if and only if $G$ is a regular graph.

Proof. For the real positive numbers $p_{1}, p_{2}, \ldots, p_{n}, a_{1}, a_{2}, \ldots, a_{n}$ and $b_{1}, b_{2}, \ldots, b_{n}$ with the property

$$
\sum_{i=1}^{n} p_{i}=1, \quad 0<r \leq a_{i} \leq R<+\infty, \quad i=1,2, \ldots, n
$$


Cerone and Dragomir [9] have proved the following inequality

$$
\left|\sum_{i=1}^{n} p_{i} a_{i} b_{i}-\sum_{i=1}^{n} p_{i} a_{i} \sum_{i=1}^{n} p_{i} b_{i}\right| \leq \frac{R-r}{2} \sum_{i=1}^{n} p_{i}\left|b_{i}-\sum_{i=1}^{n} p_{i} b_{i}\right| .
$$

For $p_{i}=\frac{1}{n}, a_{i}=b_{i}=d_{i}, i=1,2, \ldots, n, R=d_{1}$ and $r=d_{n}$, the above inequality transforms into

$$
\frac{1}{n} \sum_{i=1}^{n} d_{i}^{2}-\frac{1}{n^{2}}\left(\sum_{i=1}^{n} d_{i}\right)^{2} \leq \frac{d_{1}-d_{n}}{2 n} \sum_{i=1}^{n}\left|d_{i}-\frac{2 m}{n}\right|
$$

i.e.

$$
\frac{1}{n} M_{1}-\frac{4 m^{2}}{n^{2}} \leq \frac{d_{1}-d_{n}}{2 n} \sum_{i=1}^{n}\left|d_{i}-\frac{2 m}{n}\right| \text {. }
$$

From the above inequality we obtain

$$
\frac{4 m^{2}}{n^{2}} \operatorname{Irr}_{E}(G)^{2} \leq \frac{d_{1}-d_{n}}{2 n} \operatorname{Irr}_{N}(G)
$$

Based on the Lagrange's identity (see for example [32]) we have

$$
\begin{aligned}
& \frac{4 m^{2}}{n^{2}} \operatorname{Irr}_{E}(G)^{2}=\frac{1}{n^{2}}\left(n M_{1}-4 m^{2}\right)=\frac{1}{n^{2}}\left(n \sum_{i=1}^{n} d_{i}^{2}-\left(\sum_{i=1}^{n} d_{i}\right)^{2}\right)= \\
= & \frac{1}{n^{2}} \sum_{1 \leq i<j \leq n}\left(d_{i}-d_{j}\right)^{2} \geq \frac{1}{n^{2}}\left(\sum_{i=2}^{n-1}\left(\left(d_{1}-d_{i}\right)^{2}+\left(d_{i}-d_{n}\right)^{2}\right)+\left(d_{1}-d_{n}\right)^{2}\right) .
\end{aligned}
$$

Now according to Jennsen's inequality (see for example [31]) from the above inequality we have that

$$
\frac{4 m^{2}}{n^{2}} \operatorname{Irr}_{E}(G)^{2} \geq \frac{1}{n^{2}}\left(\sum_{i=2}^{n-1} \frac{\left(d_{1}-d_{n}\right)^{2}}{2}+\left(d_{1}-d_{n}\right)^{2}\right)=\frac{1}{2 n}\left(d_{1}-d_{n}\right)^{2} .
$$

From the above we obtain that the following is valid

$$
d_{1}-d_{n} \leq \frac{2 \sqrt{2} m}{\sqrt{n}} \operatorname{Irr}_{E}(G)
$$

Left side of the inequality (19) is obtained according to (20) and (21).

Right-hand part of inequality (19) is obtained from the Cauchy inequality for $b_{i}=1$ and $a_{i}=\left|d_{i}-\frac{2 m}{n}\right|, i=1,2, \ldots, n$ (see for example [32])

$$
\left(\sum_{i=1}^{n} a_{i} b_{i}\right)^{2} \leq \sum_{i=1}^{n} a_{i}^{2} \sum_{i=1}^{n} b_{i}
$$


Corollary 2 For every graph $G, G \in \Gamma(G)$, the following inequality is valid

$$
d_{n} \operatorname{Irr}_{g}(G) \leq \operatorname{Irr}_{N}(G) \leq n d_{n} \sqrt{\alpha(n)} \operatorname{Irr}_{g}(G) .
$$

Equalities hold if and only if $G$ is regular.

Corollary 3 For every graph $G, G \in \Gamma(G)$, the following inequalities are valid

$$
\operatorname{Irr}_{N}(G) \leq \sqrt{2 m\left(n d_{1}-2 m\right)}
$$

and

$$
\operatorname{Irr}_{N}(G) \leq \sqrt{\frac{m(n-2)(n(n-1)-2 m)}{n-2}}, \quad n \geq 3 .
$$

Equality in (22) holds if and only if $G$ is regular, whereas in (23) if $G$ is a complete graph, $G=K_{n}$.

Proof. The inequality (22) is obtained from the right-hand side of (19) and inequality $M_{1} \leq$ $2 m d_{1}$. The inequality (23) is obtained from the right-hand side of (19) and the inequality

$$
M_{1} \leq m\left(\frac{2 m}{n-1}+n-2\right),
$$

proven in [7].

Theorem 3 For every graph $G, G \in \Gamma(G)$, that satisfies Zagreb indices inequality, the following inequalities are valid

$$
\operatorname{Irr}_{I S}(G)^{3} \geq \operatorname{Irr}_{E}(G)^{2} \quad \text { and } \quad \operatorname{Irr}_{I Z}(G)^{4} \geq \operatorname{Irr}_{1}(G)^{3} .
$$

Equalities hold if and only if $G$ is regular.

Proof. Since the graph $G$ satisfies Zagreb indices inequality, then according to (1) and (2) we have that

$$
M_{2} \geq \frac{m}{n} M_{1} \quad \text { and } \quad E M_{2} \geq \frac{2 m-n}{n} E M_{1} .
$$

From the above inequalities we obtain

$$
\operatorname{Irr}_{I S}(G)^{3}=\frac{n^{2} M_{2}}{4 m^{3}}-1 \geq \frac{n^{2} m M_{1}}{4 m^{3} n}-1=\frac{n M_{1}}{4 m^{2}}-1=\operatorname{Irr}_{E}(G)^{2},
$$

and

$$
\operatorname{Irr}_{I Z}(G)^{4}=\frac{n^{3} E M_{2}}{4 m(2 m-n)^{3}}-1 \geq \frac{n^{2} E M_{1}}{4 m(2 m-n)^{2}}-1=\operatorname{Irr}_{1}(G)^{3},
$$

which had to be proved. 
The following theorem gives a relationship between irregularity measures $\operatorname{Irr}_{E}(G)$ and $\operatorname{Irr}^{(3)}(G)$.

Theorem 4 For every graph $G, G \in \Gamma(G)$, the following inequalities are valid

$$
\begin{aligned}
& \sqrt[3]{\operatorname{Irr}_{E}(G)^{2}\left(\operatorname{Irr}_{E}(G)^{2}+2\right)} \leq \operatorname{Irr} r^{(3)}(G) \leq \\
& \leq \sqrt[3]{\operatorname{Irr}_{E}(G)^{2}\left(\operatorname{Irr}_{E}(G)^{2}+2\right)+\frac{n^{2} d_{n}^{2} \beta(S)}{4 m^{2}} \operatorname{Irr}_{g}(G)^{2}},
\end{aligned}
$$

where

$$
\beta(S)=\frac{1}{2 m} \sum_{i \in S} d_{i}\left(1-\frac{1}{2 m} \sum_{i \in S} d_{i}\right),
$$

and $S$ is a subset of $I_{n}=\{1,2, \ldots, n\}$ which minimizes the expression

$$
\left|\sum_{i \in S} d_{i}-m\right|
$$

Equalities hold if and only if $G$ is regular.

Proof. For $p_{i}=d_{i}, i=1,2, \ldots, n$ the inequality (17) becomes (25). Let $S$ be a subset of $I_{n}=\{1,2, \ldots, n\}$ which minimizes the expression (25). Then for $p_{i}=a_{i}=b_{i}=d_{i}$, $i=1,2, \ldots, n, R_{1}=R_{2}=d_{1}$ and $r_{1}=r_{2}=d_{n}$, the inequality (16) becomes

$$
\sum_{i=1}^{n} d_{i} \sum_{i=1}^{n} d_{i}^{3}-\left(\sum_{i=1}^{n} d_{i}^{2}\right)^{2} \leq\left(d_{1}-d_{n}\right)^{2} \sum_{i \in S} d_{i}\left(\sum_{i=1}^{n} d_{i}-\sum_{i \in S} d_{i}\right),
$$

i.e.

$$
2 m F_{1}-M_{1}^{2} \leq 4 m^{2} \beta(S)\left(d_{1}-d_{n}\right)^{2} .
$$

If we multiply the above inequality by $\frac{n^{2}}{16 m^{4}}$ it transforms into

$$
\frac{n^{2}}{8 m^{3}} F_{1}-\left(\frac{n M_{1}}{4 m^{2}}\right)^{2} \leq \frac{n^{2} \beta(S) d_{n}^{2}}{4 m^{2}}\left(\frac{d_{1}}{d_{n}}-1\right)^{2}
$$

wherefrom we obtain right-hand part of (24).

For $\alpha=3$ the inequality ( 8 ) becomes

$$
F_{1} \geq \frac{M_{1}^{2}}{2 m}
$$


According to the above we have that the following is valid

$$
\frac{n^{2} F_{1}}{8 m^{3}}-1 \geq\left(\frac{n M_{1}}{4 m}\right)^{2}-1
$$

wherefrom we obtain the result of the theorem.

Corollary 4 For every graph $G, G \in \Gamma(G)$, the following inequality is valid

$$
\operatorname{Irr}^{(3)}(G) \leq \sqrt[3]{\operatorname{Irr}_{E}(G)^{2}\left(\operatorname{Irr}_{E}(G)^{2}+2\right)+\frac{n^{2} d_{n}^{2}}{16 m^{2}} \operatorname{Irr}_{g}(G)^{2}}
$$

Equality holds if and only if $G$ is regular.

Proof. According to the arithmetic-geometric mean inequality for real numbers (see for example [32]) we have that for each set $S, S \subseteq I_{n}$, holds $\beta(S) \leq \frac{1}{4}$. Now the inequality (26) can be obtained from the right-hand part of (24).

\section{References}

[1] H. Abdo, S. Brandt, D. Dimitrov, The total irregularity of a graph, Discr. Math. Theor. Comput. Sci., 16 (2014), 201-206.

[2] H. Abdo, D. Dimitrov, T. Reti, D. Stevanović, Estimating the spectral radius of a graph by the second Zagreb index, MATCH Commun. Math. Comput. Chem., 72 (2014), 741-751.

[3] M. O. Albertson, The irregularity of a graph, Ars Comb., 46 (1997), 219-225.

[4] D. AndRICA, C. BADEA, Grüs inequality for positive linear functionals, Period. Math. Hungar., 19 (2) (1988), 155-167.

[5] F. K. BELL, A note on the irregularity of graphs, Linear Algebra Appl., 161 (1992), 45-54.

[6] N. BIGgS, Algebraic Graph Theory, $2^{\text {nd }}$ Edition, Cambridge Univ. Press, Cambridge, 1993.

[7] D. DE CAEN, An upper bound on the sum of squares of degrees in a graph, Discrete Math., 185 (1-3), 245-248.

[8] G. CAPOROSSI, P. HANSEN, Variable neighbourhood search extremal graphs. 1. The autographix system, Discrete Math. 212 (2000) 29-44.

[9] P. CERone, S. S. Dragomir, A refinement of the Grüss inequality and applications, Tamkang J. Math., 38, 1 (2007), 34-49.

[10] F. R. K. Chung, Spectral Graph Theory, Am. Math. Soc., Providence, 1997. 
[11] L. Collatz, U. Sinogowitz, Spektren endlicher Graphen, Abh. Math. Sem. Univ Hamburg, 21 (1957), 63-77.

[12] K. CH. Das, K. XU, J. NAM, Zagreb indices of graphs, Front. Math. China, 10 (3) (2015), 5005-5012.

[13] N. DE, Some bounds of reformulated Zagreb indices, Appl. Math. Sci., 6 (101) (2012), 50055012.

[14] C. S. EDWARDS, The largest vertex degree sum for a triangle in a graph, Bull. London Math. Soc., 9 (1977), 203-208.

[15] C. ElPhick, P. Wocjan, New measures of graph irregularity, El. J. Graph. Theory Appl., 2 (1) (2014), 52-65.

[16] G. H. FATH-TABAR, Old and new Zagreb indices of graphs, MATCH Commun. Math. Comput. Chem., 65 (2011), 79-84.

[17] B. Furtula, I. Gutman, A forgotten topological index, J. Math. Chem., 53 (2015), 11841190.

[18] B. Furtula, I. Gutman, S. Ediz, On difference of Zagreb indices, Discr. Appl. Math., 178 (2014), 83-88.

[19] F. GOLDBERG, Spectral radius minus average degree: a better bound, arXiv: $1407.4285 \mathrm{v} 1$ [math.co] 16 July, 2014.

[20] I. Gutman, A degree-based topological indices, Croat. Chem. Acta, 86 (2013), 351-361.

[21] I. Gutman, B. Furtula, C. ElPhick, The new/old vertex-degree-based topological indices, MATCH Commun. Math. Comput. Chem., 72 (2014), 617-632.

[22] I. Gutman, B. Furtula, Ž. Kovijanić Vukićević, G. Popivoda, On Zagreb indices and coindices, MATCH Commun. Math. Comput. Chem., 74 (2015), 5-16.

[23] I. Gutman, N. Trinajstić, Graph theory and molecular orbitas. Total $\pi$-electron energy of alternant hydrocarbons, Chem. Phys. Lett., 17 (1972), 535-538.

[24] A. HAMZEH, T. RETI, An analogue of Zagreb index inequality obtained from graph irregularity measures, MATCH Commun. Math. Comput. Chem.,72 (2014), 669-683.

[25] A. Ilić, D. STEvanović, On comparing Zagreb indices, MATCH Commun. Math. Comput. Chem., 62 (2009), 681-687.

[26] A. ILIĆ, B. Zhou, On reformulated Zagreb indices, Discr. Appl. Math., 160 (2012), 204-209.

[27] A. MilićEvić, S. Nikolić, On variable Zagreb idices, Croat. Chem. Acta, 77 (2004), 97101.

[28] A. MilićEvić, S. Nikolić, N. Trinajstić, On reformulated Zagreb indices, Mol. Divers., 8 (2004), 393-399.

[29] E. Milovanović, E. Glogić, I. Milovanović, M. Cvjetković, Irregularity measures of graphs, Sci. Publ. State Univ. Novi Pazar, Ser. App. Math. Inform. Mechan., 7 (2), (2015), $105-116$.

[30] I. Ž. Milovanović, E. I. Milovanović, Discrete mathematics, Univ. Niš, 2000. (In serbian) 
[31] D. S. Mitrinović, J. E. PeČArić, A. M. Fink, Classical and new inequalities in analysis, Kluwer Academic Publishers, Dordrecht, 1993.

[32] D. S. Mitrinović, P. M. Vasić, Analytic inequalities, Springer Verlag, Berlin, 1970.

[33] J. V. S. NAGY, Uber algebraische Gleichungen mit lauter reellen Wurzeln, Jahresbericht der deutschen mathematiker-Vereingung, 27 (1918), 37-43.

[34] V. Nikiforov, Eigenvalues and degree deviation in graphs, Linear Algebra Appl., 414 (2006), 347-360.

[35] S. Nikolić, G. KovačEvić, A. MiLićEvić, N. Trinajstić, The Zagreb indices 30 years after, Croat. Chem. Acta, 76 (2003), 113-124.

[36] T. Popoviciu, Sur les equations algebriques ayant toutes leurs raeines reelles, Mathematica, 9 (1935), 129-145.

[37] J. RADA, R. CRUZ, Vertex-degree-based topological indices over graphs, MATCH Commun. Math. Comput. Chem. 72 (2014) 603-616.

[38] T. RÉTI, I. FelDE, Novel Zagreb indices-based inequalities with particular regard to semiregular and generalized semiregular graphs, ArXiv.org/pdf/1509.08015.

[39] R. Sharma, M. GUPTA, G. KAPOOR, Some better bounds on the variance with applications, J. Math. Ineq., (2010), 4 (3) (2010), 355-363.

[40] R. TODESCHINI, V. CONSONNI, Handbook of molecular descriptors for chemoinformatics, Wiley-VCH, Weinheim, 2000.

[41] R. TOdeschinI, V. Consonni, Molecular descriptors for chemoinformatics, Wiley-VCH, Weinheim, 2009.

[42] N. Trinajstić, Chemical Graph Theory, 2nd revised ed., CRC Press, Boca Ration, FL, 1992.

[43] D. VukičEvić, I. Gutman, B. Furtula, V. Andova, D. Dimitrov, Some observations on comparing Zagreb indices, MATCH Commun. Math. Comput. Chem., 66 (2011), 627-645.

[44] Y. S. Yoon, J. K. Kim, A relationship between bounds on the sum of squares of degrees of a graph, J. Appl. Math Comput., 21 (2006), 233-238.

[45] B. Zhou, N. Trinajstić, Some properties of the reformulated Zagreb indices, J. Math. Chem., 48 (2010), 714-719. 